\title{
Actinic flux and photolysis in water droplets: Mie calculations and geometrical optics limit
}

\author{
B. Mayer ${ }^{1, *}$ and S. Madronich ${ }^{2}$ \\ ${ }^{1}$ Deutsches Zentrum für Luft- und Raumfahrt (DLR), Oberpfaffenhofen, Germany \\ ${ }^{2}$ National Center for Atmospheric Research (NCAR), Boulder, CO, USA \\ *during this research at: National Center for Atmospheric Research (NCAR), Boulder, CO, USA \\ Received: 4 June 2004 - Published in Atmos. Chem. Phys. Discuss.: 3 August 2004 \\ Revised: 13 November 2004 - Accepted: 15 November 2004 - Published: 22 November 2004
}

\begin{abstract}
Photolysis of water-soluble components inside cloud droplets by ultraviolet/visible radiation may play an important role in atmospheric chemistry. Two earlier studies have suggested that the actinic flux and hence the photolysis frequency within spherical droplets is enhanced relative to that in the surrounding air, but have given different values for this enhancement. Here, we reconcile these discrepancies by noting slight errors in both studies that, when corrected, lead to consistent results. Madronich (1987) examined the geometric (large droplet) limit and concluded that refraction leads to an enhancement factor, averaged over all incident directions, of 1.56 . However, the physically relevant quantity is the enhancement of the average actinic flux (rather than the average enhancement factor) which we show here to be 1.26 in the geometric limit. Ruggaber et al. (1997) used Mie theory to derive energy density enhancements slightly larger than 2 for typical droplet sizes, and applied these directly to the calculation of photolysis rates. However, the physically relevant quantity is the actinic flux (rather than the energy density) which is obtained by dividing the energy density by the refractive index of water, 1.33. Thus, the Mie-predicted enhancement for typical cloud droplet sizes is in the range 1.5 , only coincidentally in agreement with the value originally given by Madronich. We also investigated the influence of resonances in the actinic flux enhancement. These narrow spikes which are resolved only by very high resolution calculations are orders of magnitude higher than the intermediate values but contribute only little to the actinic flux enhancement when averaged over droplet size distributions. Finally, a table is provided which may be used to obtain the actinic flux enhancement for the photolysis of any dissolved species.
\end{abstract}

Correspondence to: $\mathrm{B}$. Mayer

(bernhard.mayer@dlr.de)

\section{Introduction}

Photolysis inside cloud droplets may be important for atmospheric chemistry (Chameides and Davis, 1982; Lelieveld and Crutzen, 1991; Jacob, 2000). According to Jacob (2000), heterogeneous chemistry involving reactions in aerosol particles and cloud droplets may affect ozone concentrations in a number of ways including production and loss of $\mathrm{HO}_{\mathrm{x}}$ and $\mathrm{NO}_{\mathrm{x}}$, direct loss of ozone, and production of halogen radicals. Photolysis frequencies are determined by the actinic flux $F_{0}$ (Madronich, 1987). Clouds and aerosols are known to alter the actinic flux by scattering and absorption (Madronich, 1987; Ruggaber et al., 1994; Junkermann, 1994; Lantz et al., 1996; Los et al., 1997; Mayer et al., 1998; Crawford et al., 2003). Close to the cloud top large enhancements may be found while deeper into and below the cloud the actinic flux is usually reduced. An additional effect occurs for photolytic reactions of chemical species present in cloud water droplets: here the actinic flux is additionally altered due to refraction and diffraction. This paper addresses exclusively the droplet effect.

Several estimates of actinic fluxes within droplets have been reported in the literature. Graedel and Goldberg (1983) multiplied the gas phase actinic flux by 0.9 to account for loss by reflection at the air-water interface. Madronich (1987) showed that in the geometric limit of large droplets, the initial reflections are compensated by multiple internal reflections, and an overall enhancement in actinic flux would be expected due to refractive increases in photon pathlengths. Bott and Zdunkowski (1987) used Mie theory to show that the timeaveraged electromagnetic energy within dielectric spheres is enhanced by slightly more than 2 , with much higher values at multiple but narrow resonances. Ruggaber et al. (1997) applied the results of Bott and Zdunkowski (1987) to estimate photolysis coefficients for droplet size distributions representative of several different types of clouds, and again found a factor of ca. 2 enhancement relative to interstitial air, with negligible contributions from the resonances.

(C) 2004 Author(s). This work is licensed under a Creative Commons License. 
Here, we re-examine this issue by re-evaluating the studies of Madronich (1987) and Ruggaber et al. (1997) and resolve the apparent discrepancy between their results. We show that, due to an averaging error in the calculation, the geometric optics result of Madronich (1987) is too high (1.56 instead of 1.26). Ruggaber et al. (1997) assumed that the actinic flux is the product of the energy density $u$ and the speed of light in vacuum, $c_{0}$. However, the latter assumption is not correct. E.g. Chandrasekhar (1950) and Lenoble (1993) show that the actinic flux $F$ is the product of the energy density $u$ and the velocity $c$ of light, but the relevant quantity is the speed of light in the medium, $c=c_{0} / n$, where $n$ is the refractive index of the medium. Consequently, the enhancement of the actinic flux inside a droplet is the ratio of energy densities inside and outside the droplet divided by the refractive index of the medium. The values found by Ruggaber et al. (1997) have therefore to be divided by the refractive index of water, 1.33 , when applied to the calculation of photolysis frequencies. Here we demonstrate that geometrical optics and Mie calculations agree perfectly well in the limit of large particles taking into account both corrections.

In the following section, results of the geometrical optics calculation are compared to rigorous Mie theory. A table of enhancement factors is presented, to be used in aqueousphase chemistry calculations. The relevance for the application of the results by Ruggaber et al. (1997) is discussed in the conclusions. Appendix A explains the relationship between the actinic flux and other radiative quantities, in particular the energy density $u$ which is crucial for our investigation. In Appendix B the geometrical optics calculation is presented in full detail.

\section{Calculations}

The actinic flux $F_{0}$ is defined as the integral of the radiance $L(\theta, \phi)$ over the full solid angle $4 \pi$ :

$F_{0}=\int_{4 \pi} L(\theta, \phi) d \Omega$.

Appendix A explains how the actinic flux is related to other quantities of the radiation field, in particular the energy density $u$ :

$u=\frac{1}{c} F_{0}$,

where $c$ is the speed of light in the medium. Due to the interaction between radiation and matter, the actinic flux inside a droplet differs from the unperturbed case. The actinic flux enhancement in a droplet can be derived from the ratio of energy densities inside and outside the droplet as by Ruggaber et al. (1997). Here we use a different but equivalent approach based on the absorption efficiency $Q_{\text {abs }}$. To calculate the enhancement of the actinic flux inside a droplet, we compare the average actinic flux in the medium to the actinic flux in the absence of the droplet. As both internal and external electromagnetic fields are perturbed by the presence of a droplet, we adopt the terms $F_{0 \text {,perturbed for the actinic flux }}$ inside the medium and $F_{0 \text {, unperturbed }}$ for the field in absence of the droplet.

The radiant power absorbed by a droplet, $\frac{d W_{\mathrm{abs}}}{d t}$, can be expressed using the absorption efficiency $Q_{\mathrm{abs}}=\Sigma_{\mathrm{abs}} / \pi r^{2}$ where $\Sigma_{\text {abs }}$ is the absorption cross section and $\pi r^{2}$ is the geometrical cross section of the droplet with radius $r$ :

$$
\begin{aligned}
\frac{d W_{\mathrm{abs}}}{d t} & =\int L_{\text {unperturbed }}(\theta, \phi) \cdot Q_{\mathrm{abs}} \cdot \pi r^{2} d \Omega \\
& =F_{0, \text { unperturbed }} \cdot Q_{\mathrm{abs}} \cdot \pi r^{2},
\end{aligned}
$$

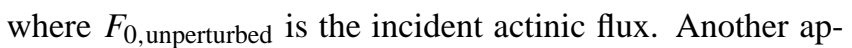
proach is to express the influence of the medium on the radiation field by introducing a perturbed actinic flux, $\overline{F_{0 \text {,perturbed }}}$, and looking at individual absorbers:

$\frac{d W_{\text {abs }}}{d t}=\overline{F_{0, \text { perturbed }}} \cdot N \cdot \sigma_{\text {abs }}$,

where $N$ is the number of absorbing molecules in the droplet, $\sigma_{\mathrm{abs}}$ is the absorption cross section of an individual absorbing molecule, and $\overline{F_{0, \text { perturbed }}}$ is the average actinic flux inside the droplet. Without loss of generality we assume there is only one absorbing species. Please note, however, that we assume that the absorbing species is distributed uniformly in the droplet. Combining Eqs. (3) and (4) the actinic flux enhancement $\eta$ is calculated as

$\eta=\frac{\overline{F_{0, \text { perturbed }}}}{F_{0, \text { unperturbed }}}=\frac{\Sigma_{\mathrm{abs}}}{N \cdot \sigma_{\mathrm{abs}}}=\frac{Q_{\mathrm{abs}} \cdot \pi r^{2}}{N \cdot \sigma_{\mathrm{abs}}}$.

This equation is easily understood: The actinic flux enhancement is simply the ratio of the absorption by the droplet (with dissolved molecules) and the absorption by the individual molecules, in absence of the droplet. In the following, the absorption efficiency of the droplet $Q_{\mathrm{abs}}$ is approximated in the geometrical optics limit and calculated using rigorous Mie theory, to determine the actinic flux enhancement $\eta$.

\subsection{Geometrical optics}

In the geometrical optics limit, individual light rays are considered independently. This approach can of course only be applied to droplets that are large compared to the wavelength of the radiation. The absorption of radiation is calculated by tracing the radiation on individual paths through the droplet and summing all contributions, see Fig. 1.

In Appendix B, the geometric optics approximation is described in detail. A numerical solution is provided for arbitrary absorption, and it is shown that in the limit of small absorption, $k_{\mathrm{abs}} \cdot r \ll 1$, the actinic flux enhancement can be evaluated analytically to yield

$\eta=n^{2} \cdot\left[1-\left(1-\frac{1}{n^{2}}\right)^{3 / 2}\right]$. 


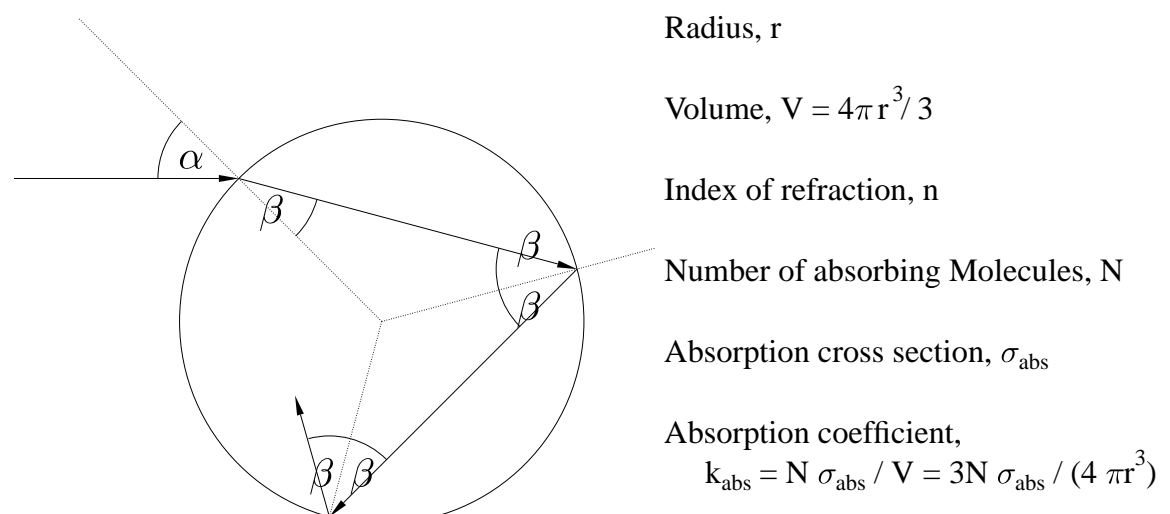

Wavelength, $\lambda$

Size parameter, $\mathrm{x}=2 \pi \mathrm{r} / \lambda$

Fig. 1. Schematics of a droplet. In the geometrical optics limit, the radiation is traced along individual paths which are considered independent. Refraction is described by Snell's law and reflection follows Fresnel's equations.

In the case of water, the refractive index $n$ varies between 1.35 at $300 \mathrm{~nm}$ and 1.33 at $800 \mathrm{~nm}$ (Hale and Querry, 1973), or 1.37 at $300 \mathrm{~nm}$ and 1.33 at $800 \mathrm{~nm}$ (Wiscombe, 1994); the temperature dependence between -10 and $50^{\circ} \mathrm{C}$ is negligible (Harvey et al., 1998). For a value of 1.33 the corresponding actinic flux enhancement is 1.26 . Figure 2 shows the actinic flux enhancement as a function of the product $k_{\mathrm{abs}} \cdot r$. Up to $k_{\text {abs }} \cdot r=10^{-3}$ absorption can obviously be neglected. For a typical cloud droplet size of $10 \mu \mathrm{m}$, this corresponds to an absorption coefficient of $k_{\mathrm{abs}}=100 \mathrm{~m}^{-1}$, a large number. Pure water at $305 \mathrm{~nm}$ has an absorption coefficient of about $0.3 \mathrm{~m}^{-1}$, see below. In addition, absorption due to dissolved molecules has to be considered, e.g. ozone. According to Yin et al. (2001), the concentration of ozone is in Henry's law equilibrium and can hence be calculated by

$n_{\mathrm{O}_{3}, \text { liq }}=H_{\mathrm{O}_{3}} \cdot p_{\mathrm{O}_{3}}=1.8 \cdot 10^{11} \mathrm{~cm}^{-3}$,

where $n_{\mathrm{O}_{3} \text {, liq }}$ is the ozone concentration in the liquid phase, $H_{\mathrm{O}_{3}}=1.1 \cdot 10^{-7} \mathrm{~mol} \cdot \mathrm{kg}^{-1} \cdot \mathrm{Pa}^{-1}$ is Henry's law constant for ozone (Kosak-Channing and Helz, 1983), and $p_{\mathrm{O}_{3}}=2.7 \cdot 10^{-3} \mathrm{~Pa}$ is the partial pressure of ozone in the boundary layer of the US standard atmosphere. At a wavelength of $305 \mathrm{~nm}$ where the maximum of the contribution to the $\mathrm{O}\left({ }^{1} D\right)$ photolysis frequency usually occurs, the absorption cross section of ozone is $\sigma=2 \cdot 10^{-19} \mathrm{~cm}^{2}$; together with the above calculated $n_{\mathrm{O}_{3} \text {, liq }}$ this results in an absorption coefficient of $k_{\mathrm{abs}}=n_{\mathrm{O}_{3} \text {, liq }} \cdot \sigma=3.6 \cdot 10^{-6} \mathrm{~m}^{-1}$ which is seven orders of magnitude below the limiting value. Hence, absorption by pure water itself as well as by the dissolved component may be safely ignored for the calculation of ultraviolet actinic fluxes in cloud water droplets.

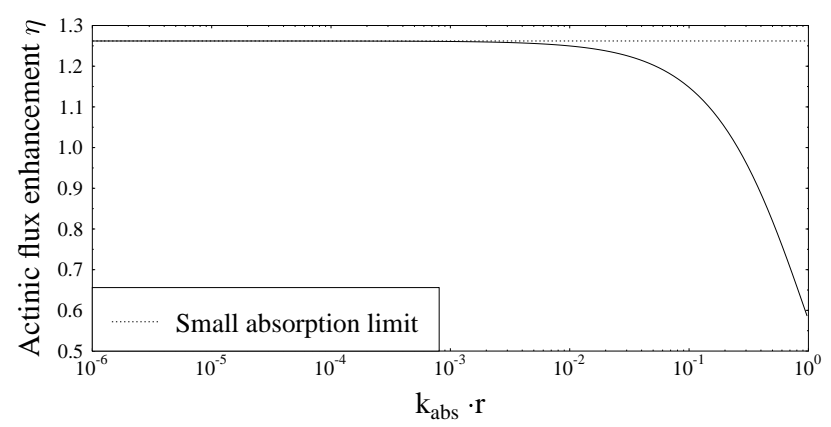

Fig. 2. Actinic flux enhancement as a function of absorption in the geometrical optics limit.

Madronich (1987) also calculated the enhancement of the actinic flux inside a water droplet in Sect. 3.3 of his paper. The four assumptions presented there are correct, as is the enhancement factor for any incident ray. However, at the end Madronich (1987) averages the enhancement factor over all incident rays, while the physically relevant quantity is the ratio of the perturbed and unperturbed actinic fluxes, each individually averaged. If this modification is introduced in the last step of Madronich's calculations, the final result is in agreement with the geometric limit found here, see Eq. (6). The correct calculation is presented in Appendix B.

\subsection{Mie calculations}

Calculations for droplets which are not much larger than the wavelength of the radiation require application of rigorous Mie theory. In order to calculate the actinic flux en- 


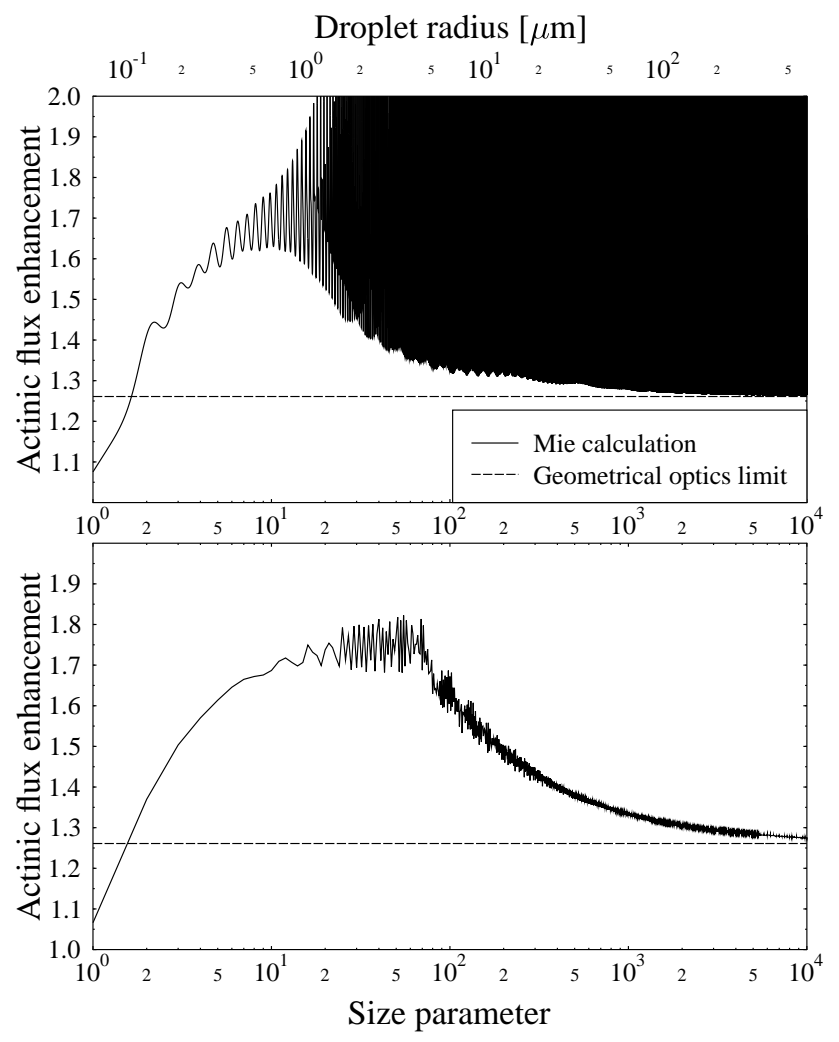

Fig. 3. (Top) Mie calculation of the actinic flux enhancement with MIEV and geometrical optics result. The upper $\mathrm{x}$-axis shows the corresponding droplet radius for a wavelength of $400 \mathrm{~nm}$. (Bottom) Same data, but averaged over size parameter bins of width 1 .

hancement inside water droplets, two different Mie programs were employed, MIEV (Wiscombe, 1979, 1980) and BHMIE (Bohren and Huffman, 1983). Both programs provide the absorption efficiency $Q_{\text {abs }}$, which is used to infer the actinic flux enhancement $\eta$ according to Eq. (5). Introducing the complex refractive index according to Born and Wolf (2003),

$\hat{n}=n(1+i \kappa)$

with

$n \kappa=\frac{k_{\mathrm{abs}} \cdot \lambda}{4 \pi}$

into (5) and remembering that $k_{\mathrm{abs}}=N / V \cdot \sigma_{\mathrm{abs}}$ where $N / V$ is the absorber density, the actinic flux enhancement evaluates to

$\eta=\frac{3 Q_{\mathrm{abs}}}{8 \times n \kappa}$,

where $x=2 \pi r / \lambda$ is the size parameter. Comparing this result with Eq. (16) of Bott and Zdunkowski (1987),

$\frac{u_{\text {perturbed }}}{u_{\text {unperturbed }}}=n \cdot \frac{3 Q_{\mathrm{abs}}}{8 \times n \kappa}$

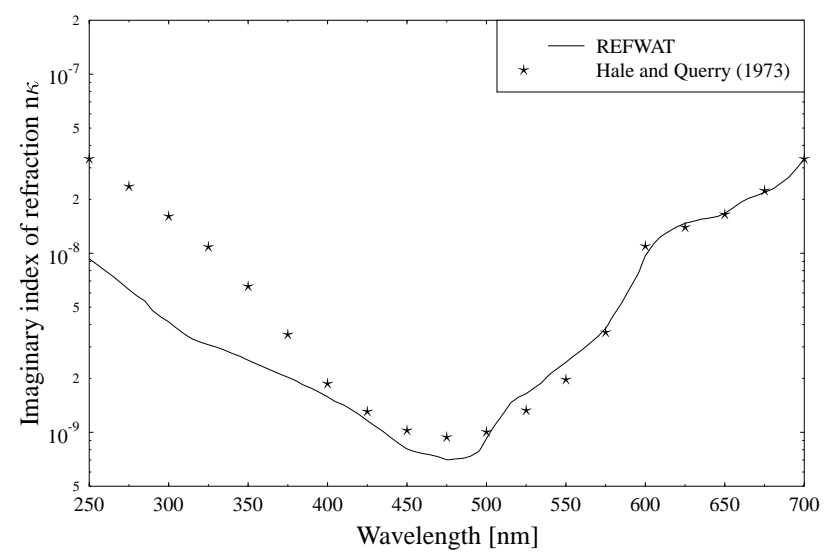

Fig. 4. Imaginary refractive index of water as provided by REFWAT (Wiscombe, 1994) in comparison to data from Hale and Querry (1973).

(in the limit of small absorption, $\kappa \ll 1$ ) it becomes clear that the ratio of energy densities has to be divided by the real part of the refractive index of the medium, $n$, in order to get the ratio of actinic fluxes. Thus, the photolysis enhancements reported by Ruggaber et al. (1997), while for the most part correct, should be divided by the refractive index of water. In practice, this reduces their stated enhancement from about a factor of 2 to ca. 1.5, in coincidental agreement with the original value proposed by Madronich (1987) but substantially higher than the actual geometrical limit of 1.26.

Figure 3 shows the enhancement of the actinic flux, derived from a calculation of the absorption efficiency $Q_{\mathrm{abs}}$ with MIEV, according to Eq. (10). The imaginary refractive index was set to a very small value of $10^{-9}$ which is a reasonable lower boundary for pure water in the wavelength region we are interested in, see Fig. 4.

Figure 3 is a little hard to interpret, due to the limited resolution of the human eye. Looking more closely one would find that the curve generally is close to the lower envelope, and that the blackened area is caused by thousands of individual spikes, so-called resonances. Figure 5 shows as an example a particular resonance which has been investigated in detail by Ray and Bhanti (1997).

This figure has been simulated with MIEV, and the exact coincidence of the location of resonance with the value reported by Ray and Bhanti (1997) gives us confidence that MIEV captures this subtle feature correctly. The step width for the MIEV calculation was $10^{-7}$ which is obviously enough to resolve the peak. If a larger value would have been chosen for the step width, part of the peak would have been missed. Please note that the peak height in this case is $4.5 \cdot 10^{4}$ which is four orders of magnitude higher than the lower envelope of the curve (assuming a realistic imaginary refractive index of $10^{-9}$ ). Such resonances might therefore have the potential to increase the actinic flux and hence the 


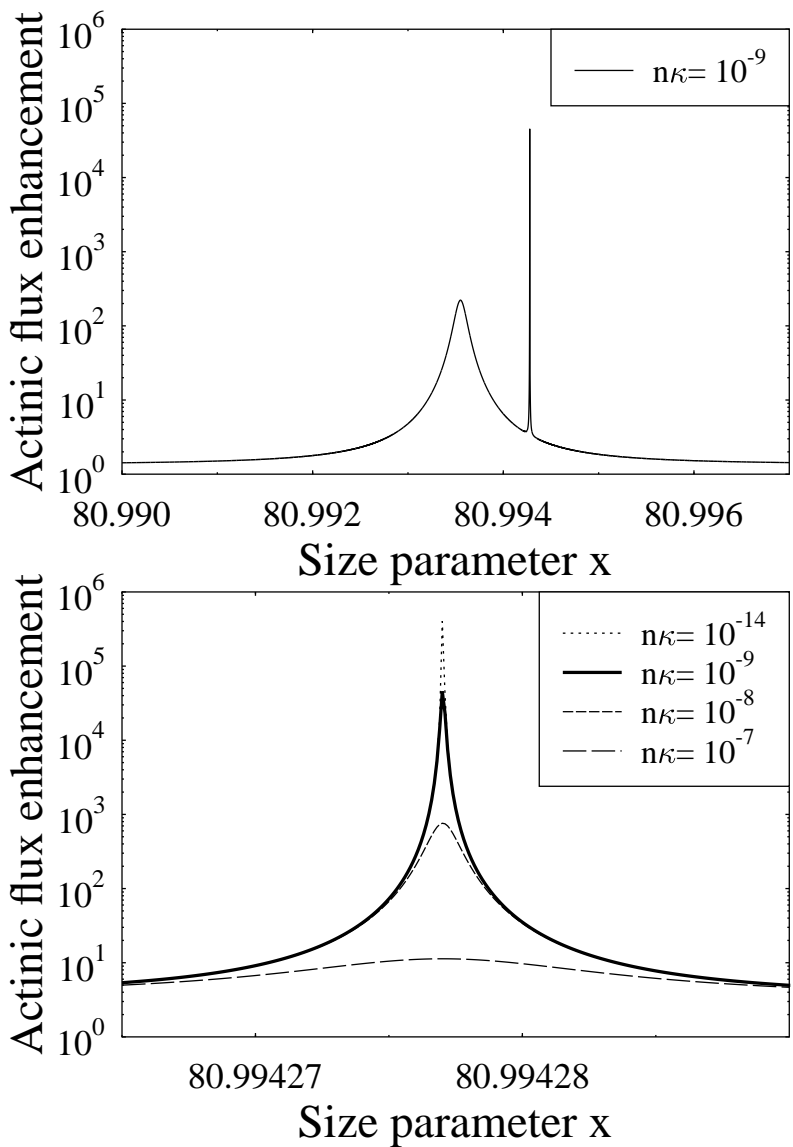

Fig. 5. (Top) Example of a spike in the actinic flux enhancement, calculated with MIEV. (Bottom) Magnification of the narrow resonance in the left image for different imaginary refractive indices.

absorption in water droplets significantly. To illustrate the relevance of the spikes we averaged the actinic flux enhancement over size parameter intervals of width 1 (bottom plot in Fig. 3). Here it is obvious that the resonances might increase the actinic flux enhancement somewhat but, for our purposes, not significantly. Figure 5 also illustrates that the amplitude of the resonances decreases rapidly with increasing absorption.

A question of particular interest is if the resonances cause problems in lower resolution calculations where the small spikes are not adequately resolved. With a step size of $10^{-7}$, years of computational time would be required on a modern PC to calculate a curve like the top plot in Fig. 3, even with the fast MIEV code (the calculation was done on a multiprocessor Linux cluster). Therefore, much lower resolutions are usually chosen. To study the influence of the resolution, we calculated the actinic flux enhancement with different step widths, $10^{-7}, 10^{-6}, 10^{-5}$, and $10^{-4}$ and integrated those over size parameter intervals of width 1 . Figure 6 shows the ratio of the results for different resolutions. We call the high-

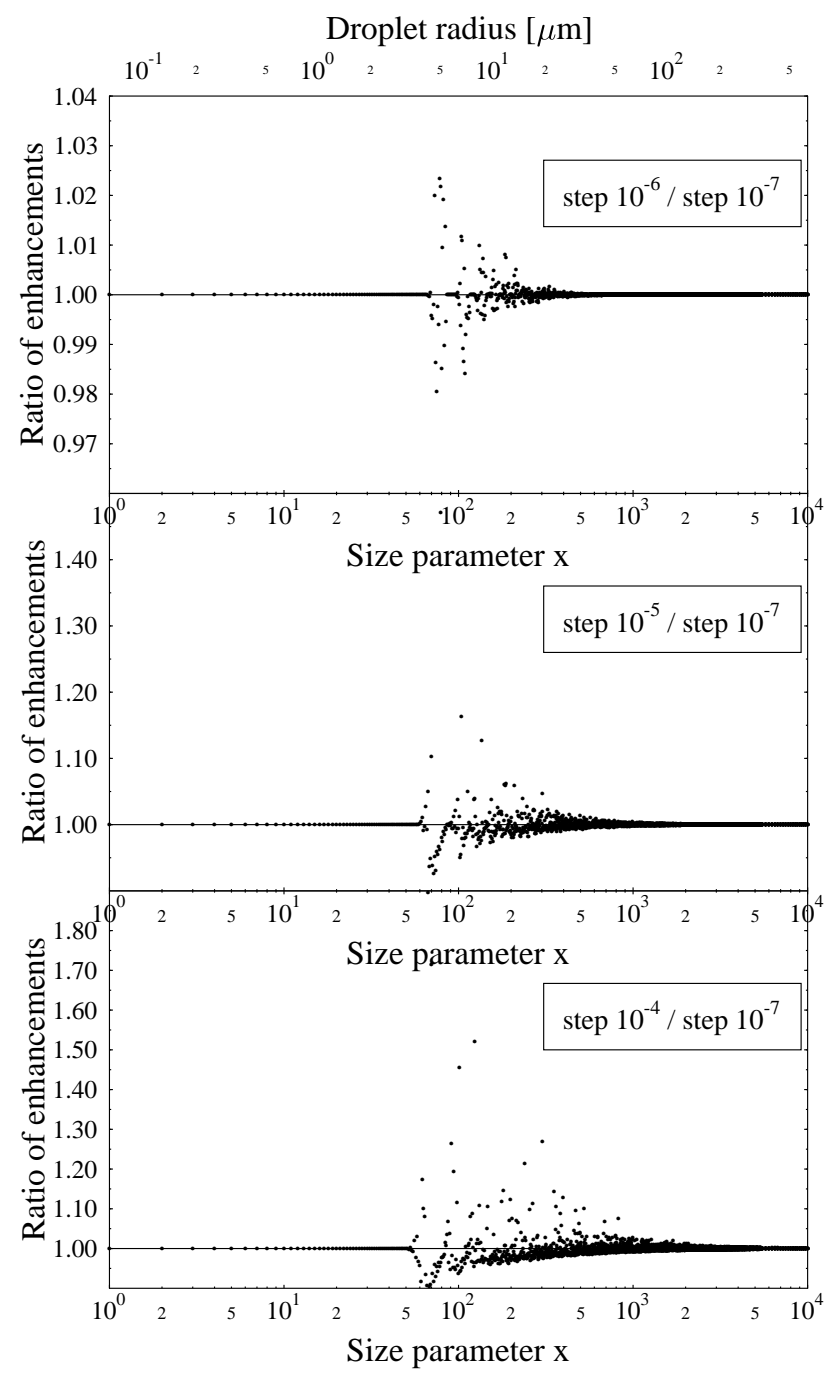

Fig. 6. Ratio of the actinic flux enhancement, calculated with different size parameter resolutions. (Top) step width $10^{-6}$ compared to step width $10^{-7}$; (middle) step width $10^{-5}$ compared to step width $10^{-7}$; (bottom) step width $10^{-4}$ compared to step width $10^{-7}$. Please note the different y-scales!

resolution result the "true value" because the resolution is high enough to fully resolve the peak. For a step width of $10^{-6}$, the difference to the true result is smaller than $\pm 3 \%$. For $10^{-5}$ the difference increases and for $10^{-4}$ a clear pattern arises: In most intervals the enhancement is underestimated because one or more resonances are missed by the low resolution calculation. In some intervals large over-estimation occurs (up to a factor of 1.8); here the low-resolution calculation accidentally hits a peak which is then "smeared out" over an interval of $10^{-4}$ and therefore contributes more than it should. On average over the whole range both effects practically cancel (the average ratio over the whole size parameter range is 0.99984 ) although locally large differences exist. For typical droplet size distributions (see e.g. Mayer et al., 
Table 1. Average enhancement factors $\bar{\eta}$ as a function of wavelength and effective droplet radius. The value $\infty$ is the geometrical optics limit which is valid for very large (rain) drops. Also shown are the real and imaginary parts of the refractive index.

\begin{tabular}{|c|c|c|c|c|c|c|}
\hline \multirow{2}{*}{$\begin{array}{c}\text { Effective } \\
\text { radius } \\
{[\mu m]}\end{array}$} & \multicolumn{6}{|c|}{$\begin{array}{c}\text { Wavelength } \\
{[\mathrm{nm}]}\end{array}$} \\
\hline & 300 & 400 & 500 & 600 & 700 & 800 \\
\hline 1.0 & 1.840 & 1.771 & 1.732 & 1.704 & 1.681 & 1.662 \\
\hline 2.0 & 1.839 & 1.795 & 1.763 & 1.741 & 1.724 & 1.710 \\
\hline 3.0 & 1.758 & 1.776 & 1.771 & 1.751 & 1.737 & 1.726 \\
\hline 4.0 & 1.679 & 1.731 & 1.757 & 1.746 & 1.738 & 1.730 \\
\hline 5.0 & 1.622 & 1.684 & 1.725 & 1.722 & 1.723 & 1.720 \\
\hline 6.0 & 1.582 & 1.644 & 1.687 & 1.690 & 1.696 & 1.698 \\
\hline 7.0 & 1.552 & 1.610 & 1.652 & 1.658 & 1.666 & 1.671 \\
\hline 8.0 & 1.527 & 1.582 & 1.622 & 1.628 & 1.637 & 1.643 \\
\hline 9.0 & 1.508 & 1.558 & 1.596 & 1.602 & 1.611 & 1.617 \\
\hline 10.0 & 1.491 & 1.538 & 1.574 & 1.579 & 1.589 & 1.594 \\
\hline 11.0 & 1.477 & 1.521 & 1.555 & 1.560 & 1.569 & 1.574 \\
\hline 12.0 & 1.465 & 1.506 & 1.539 & 1.543 & 1.552 & 1.556 \\
\hline 13.0 & 1.455 & 1.493 & 1.525 & 1.528 & 1.536 & 1.540 \\
\hline 14.0 & 1.445 & 1.482 & 1.512 & 1.514 & 1.523 & 1.526 \\
\hline 15.0 & 1.437 & 1.471 & 1.501 & 1.503 & 1.510 & 1.514 \\
\hline 16.0 & 1.430 & 1.462 & 1.491 & 1.492 & 1.500 & 1.502 \\
\hline 17.0 & 1.424 & 1.454 & 1.482 & 1.482 & 1.490 & 1.492 \\
\hline 18.0 & 1.418 & 1.446 & 1.474 & 1.474 & 1.481 & 1.483 \\
\hline 19.0 & 1.412 & 1.440 & 1.467 & 1.466 & 1.472 & 1.475 \\
\hline 20.0 & 1.407 & 1.433 & 1.460 & 1.459 & 1.465 & 1.467 \\
\hline 21.0 & 1.403 & 1.428 & 1.453 & 1.452 & 1.458 & 1.460 \\
\hline 22.0 & 1.399 & 1.423 & 1.447 & 1.446 & 1.452 & 1.453 \\
\hline 23.0 & 1.395 & 1.418 & 1.442 & 1.440 & 1.446 & 1.447 \\
\hline 24.0 & 1.391 & 1.413 & 1.437 & 1.435 & 1.440 & 1.441 \\
\hline 25.0 & 1.388 & 1.409 & 1.432 & 1.430 & 1.435 & 1.436 \\
\hline$\infty$ & 1.278 & 1.270 & 1.266 & 1.263 & 1.262 & 1.260 \\
\hline$n$ & 1.371 & 1.350 & 1.339 & 1.333 & 1.329 & 1.326 \\
\hline$n \kappa / 10^{-7}$ & 0.041 & 0.016 & 0.009 & 0.097 & 0.337 & 1.250 \\
\hline
\end{tabular}

2004), however, rather high resolution is required which confirms the results of Ruggaber et al. (1997). To some degree, spikes might be excluded using the SPIKE parameter provided by MIEV, see (Wiscombe, 1979) for more information.

Figure 3 clearly demonstrates that the Mie calculation approaches the geometrical optics limit for large size parameters, but only slowly. In particular, the average enhancement for size parameters between 1000 and 10000 is 1.298 (for all four step widths, $10^{-4}, 10^{-5}, 10^{-6}$, and $10^{-7}$ ). This is only $3 \%$ higher than the geometrical optics result of 1.26 calculated according to Eq. (10). As a final check we calculated the same quantities with BHMIE and found an average enhancement of 1.328 which is $5 \%$ higher than the geometrical optics limit. A typical radius for cloud droplets is $10 \mu \mathrm{m}$, corresponding to a size parameter of 157 at $400 \mathrm{~nm}$. At $x=157$, an enhancement of 1.54 is found which is close to the value reported by Ruggaber et al. (1997), if the latter is corrected with the refractive index. By chance, this value is very close to the 1.565 which Madronich (1987) erroneously calculated. The actual geometrical optics result, 1.26, is about $20 \%$ lower.

\subsection{Implications for atmospheric chemistry}

To allow direct application of these results in aqueous phase chemistry calculations, we determined the average enhancement factor as a function of droplet size and wavelength, by integrating the enhancement over typical cloud droplet size distributions. A gamma size distribution was chosen which is a common assumption for water clouds. The gamma distribution is characterized by the effective droplet radius

$r_{\text {eff }}=\frac{\int n_{\text {droplet }}(r) r^{3} d r}{\int n_{\text {droplet }}(r) r^{2} d r}$

and the width of the size distribution $n_{\text {droplet }}(r)$. For the definition and explanation of these quantities and some examples please refer to Mayer et al. (2004).

To calculate the average enhancement factor for a droplet size distribution we need to integrate the enhancement factor weighted by the number of molecules of interest, available at each radius. Assuming that the concentration of dissolved molecules does not depend on the droplet radius, we find

$\bar{\eta}=\frac{\int n_{\text {droplet }}(r) r^{3} \eta(r) d r}{\int n_{\text {droplet }}(r) r^{3} d r}$

where the factor $r^{3}$ considers that the number of molecules dissolved in a droplet is proportional to the droplet volume $\frac{4 \pi}{3} r^{3}$ (the factor $\frac{4 \pi}{3}$ cancels in the quotient). Table 1 presents enhancement factors for all droplet effective radii occurring in common water clouds.

The wavelength dependence of both components of the complex refractive index has been considered in the calculation: The real part $n$ varies between 1.37 at $300 \mathrm{~nm}$ and 1.33 at $800 \mathrm{~nm}$ while the imaginary part $n \kappa$ has its minimum at $500 \mathrm{~nm}\left(9.7 \cdot 10^{-10}\right)$ and assumes its maximum at $800 \mathrm{~nm}$ $\left(1.3 \cdot 10^{-7}\right)$, see also Fig. 4. In addition to the variation of the enhancement factor with particle size (Fig. 3) the wavelength dependence of the refractive index introduces an extra variability of more than $10 \%$. Similar to the single scattering properties of an ensemble of droplets which are mainly determined by the effective radius of the droplet size distribution but not by the width or shape, the enhancement factor is hardly influenced by the width of the size distribution: the calculation of Table 1 was done with a $\gamma$ of 6 which is typically used for water clouds. In comparison, using a very narrow size distribution $(\gamma=100)$, the results agreed within $3 \%$ with those for the wide distribution. As indicated in the last section, a typical droplet size for water clouds is $10 \mu \mathrm{m}$ which gives an enhancement factor of between 1.5 and 1.6, depending on the wavelength. In conclusion, Table 1 may be used for the calculation of photolysis frequencies for any species dissolved in the droplets of a water cloud. For rain 
drops it is suggested to use the geometrical optics limit, also listed in the table. We did not include typical sizes of aerosol particles (smaller than $1 \mu \mathrm{m}$ ) in the table because those exhibit a wide range of composition and hence refractive indices different from pure water.

\section{Conclusions}

The enhancement of the actinic flux inside water droplets was calculated using Mie theory and also evaluated in the geometrical optics limit. We found that the exact solution converges toward the geometrical optics limit for large size parameters and thus provides consistent solutions with both methods. For the application of photolysis frequencies in water clouds, the droplet size is typically $10 \mu \mathrm{m}$ while relevant wavelengths are between 300 and $600 \mathrm{~nm}$, resulting in size parameters of about 100-200. In this range the enhancement factor is about 1.5 which is significantly larger than the geometric limit of 1.26 . The maximum enhancement, $\approx 1.8$, occurs for somewhat smaller droplets (size parameter 10-100), and can fall below the geometric limit for size parameters smaller than unity (e.g. fine aerosols). Hence it is suggested to use exact Mie theory to avoid systematic errors, or to refer to Table 1. Ruggaber et al. (1994) have used a similar approach, however, their results have to be divided by the refractive index of water, 1.33 .

Resonant spikes may cause actinic flux enhancements of 10000 and more for certain size parameters. MIEV correctly calculates these spikes. However, when averaged over realistic droplet size distributions, these spikes contribute only little to the actinic flux enhancement of the ensemble and can therefore be safely neglected. But still, the size distribution needs to be sampled at very high resolution to avoid noise introduced by spikes which are accidentally hit in a low-resolution calculation.

As already indicated by Ruggaber et al. (1997), inhomogeneous distribution of the absorber inside the droplet may have an influence on this result. Few studies are available on this subject. Ray and Bhanti (1997) allowed inhomogeneous distributions of the absorber in the droplet, but their calculations were made for very special (resonant) conditions. Such effects, however, are beyond the scope of this paper.

\section{Appendix A: The actinic flux}

The basic quantity to describe a radiation field is the spectral radiance $L$ which is the radiant energy $d W$ in the wavelength interval $d \lambda$ that crosses the area $d A \cdot \cos \Theta$ during the time $d t$ into solid angle $d \Omega$ :

$L=\frac{d W}{d t \cdot d \lambda \cdot d A \cos \Theta \cdot d \Omega}$.

$\Theta$ is the angle between the normal to the area $d A$ and the direction $(\theta, \phi)$ and $d A \cos \Theta$ is the projection of $d A$ normal to the direction of the radiation. The net flux $F$ is defined as the net energy $d W$ which crosses an area element $d A$ in the time dt:

$F=\frac{d W}{d t \cdot d \lambda \cdot d A}$.

Combining Eqs. (14) and (15) we find for the net flux through a given area element $d A$

$F=\int_{4 \pi} L(\theta, \phi) \cdot \cos \Theta d \Omega$,

Note that the net flux is simply the difference between the incoming and outgoing irradiances. For later calculations we also need the net flux vector $\boldsymbol{F}$ whose components are defined as

$F_{\mathrm{x}, \mathrm{y}, \mathrm{z}}=\int_{4 \pi} L(\theta, \phi) \cdot\left(\boldsymbol{s} \cdot \boldsymbol{e}_{\mathrm{x}, \mathrm{y}, \mathrm{z}}\right) d \Omega$,

where $\boldsymbol{s}$ is a unit vector with direction $(\theta, \phi)$ and $\boldsymbol{e}_{\mathrm{x}, \mathrm{y}, \mathrm{z}}$ are the unity vectors in the $x, y$, and $z$ directions.

In contrast to the net flux, the actinic flux $F_{0}$ is defined as the integral of the radiance over $4 \pi$ :

$F_{0}=\int_{4 \pi} L(\theta, \phi) d \Omega$.

To see the usefulness of this quantity we need the radiative transfer equation (Chandrasekhar, 1950),

$$
\frac{d L}{d s}=-k_{\mathrm{ext}} \cdot L+\frac{k_{\mathrm{sca}}}{4 \pi} \int_{4 \pi} p\left(\theta^{\prime}, \phi^{\prime}, \theta, \phi\right) L\left(\theta^{\prime}, \phi^{\prime}\right) d \Omega^{\prime},
$$

where $k_{\text {ext }}$ is the extinction coefficient, $k_{\text {sca }}$ is the scattering coefficient, and $p\left(\theta^{\prime}, \phi^{\prime}, \theta, \phi\right)$ is the scattering phase function which is the probability that radiation coming from direction $\left(\theta^{\prime}, \phi^{\prime}\right)$ is scattered into direction $(\theta, \phi)$, normalized to $4 \pi$. The first term on the right side is the extinction of radiation while the second describes the scattering of radiation into the direction $s$. Please note that Eq. (19) includes neither thermal emission nor inelastic scattering. Both can be safely neglected in the calculation of photolysis frequencies. The left side of Eq. (19) is a directional derivative which can also be written as $s \cdot \nabla$ where $s$ is a unit vector. Integrating Eq. (19) over the solid angle $d \Omega$ we get

$$
\begin{aligned}
& \int_{4 \pi} s \cdot \nabla L d \Omega=-k_{\mathrm{ext}} \cdot \int_{4 \pi} L d \Omega+ \\
& \frac{k_{\mathrm{sca}}}{4 \pi} \int_{4 \pi} L\left(\theta^{\prime}, \phi^{\prime}\right) \int_{4 \pi} p\left(\theta^{\prime}, \phi^{\prime}, \theta, \phi\right) d \Omega d \Omega^{\prime} .
\end{aligned}
$$

The left side evaluates to

$$
\begin{gathered}
\int_{4 \pi} \boldsymbol{s} \cdot \nabla L d \Omega=\int_{4 \pi}\left(s_{\mathrm{X}} \frac{\partial L}{\partial x}+s_{\mathrm{y}} \frac{\partial L}{\partial y}+s_{\mathrm{z}} \frac{\partial L}{\partial z}\right) d \Omega= \\
=\frac{\partial}{\partial x} \int_{4 \pi} L \cdot\left(\boldsymbol{s} \cdot \boldsymbol{e}_{\mathrm{X}}\right) d \Omega+\frac{\partial}{\partial y} \int_{4 \pi} L \cdot\left(\boldsymbol{s} \cdot \boldsymbol{e}_{\mathrm{y}}\right) d \Omega+ \\
\frac{\partial}{\partial z} \int_{4 \pi} L \cdot\left(\boldsymbol{s} \cdot \boldsymbol{e}_{\mathrm{z}}\right) d \Omega=\nabla \boldsymbol{F},
\end{gathered}
$$


while the integral of the phase function on the right side of Eq. (20) simply gives $4 \pi$. Combining these, we find

$\nabla \boldsymbol{F}=-\left(k_{\mathrm{ext}}-k_{\mathrm{sca}}\right) \cdot \int_{4 \pi} L d \Omega=-k_{\mathrm{abs}} \cdot F_{0}$.

Hence, the actinic flux is the divergence of the net flux divided by the absorption coefficient. If we recall the meaning of the divergence using Gauss' theorem,

$$
\int_{V} \nabla \boldsymbol{F} d V=\int_{\partial V} \boldsymbol{F} \cdot \boldsymbol{n} d A,
$$

we find that $\nabla \boldsymbol{F}$ equals the net energy per unit time that enters the volume $V$ because the right side of Eq. (22) is the net energy transported across the volume boundary $\partial V$. Under steady state conditions, this number must equal the absorption, for which reason the absorbed radiant power can be expressed as

$\frac{d W_{\mathrm{abs}}}{d t \cdot d \lambda \cdot d V}=-\nabla \boldsymbol{F}=k_{\mathrm{abs}} \cdot F_{0}$.

If we divide by the photon energy $\frac{h c}{\lambda}$ and the absorber density $n_{\text {abs}}$, and integrate over wavelength, we find

$j_{\mathrm{abs}}=\int \frac{k_{\mathrm{abs}}}{n_{\mathrm{abs}}} \cdot \frac{F_{0}}{\frac{h c}{\lambda}} d \lambda=\int \sigma_{\mathrm{abs}} \cdot \frac{F_{0}}{\frac{h c}{\lambda}} d \lambda$,

where $j_{\mathrm{abs}}$ is the number of photons absorbed per unit time by a single absorber molecule and $\sigma_{\mathrm{abs}}$ is the absorption cross section of the individual molecule. Please note that $F_{0} / \frac{h c}{\lambda}$ is simply the actinic flux expressed in photons $/\left(\mathrm{m}^{2} \mathrm{~nm} \mathrm{~s}\right)$. Introducing the quantum yield $\Phi$ which gives the propability that a certain reaction will actually happen once a photon is absorbed, we finally find:

$j=\int \sigma_{\mathrm{abs}} \cdot \Phi \cdot F_{0} / \frac{h c}{\lambda} d \lambda$,

where $j$ is the photolysis frequency. This is the well-known formula used to calculate photolysis frequencies (Madronich, 1987). Equation (23) can of course be applied to individual reactions by using absorption cross section and quantum yield for specific molecules, while for the determination of the actinic flux in Eq. (21) the total absorption coefficient is the relevant quantity.

As a last step, we want to relate the actinic flux $F_{0}$ to the energy density $u$ of the radiation field:

$u=\frac{d W}{d \lambda \cdot d V}$.

For this purpose, consider a cylinder with cross section $d A$ and length $d l$, with the radiation entering perpendicular to the front face. The energy that enters the cylinder is

$d W=L \cdot d A \cdot d \Omega \cdot d \lambda \cdot d t$,

where $d t$ is the time required to traverse the cylinder with $d t=d l / c$ where $c$ is the speed of light in the medium. On the other hand, according to Eq. (24) the energy can also be expressed as

$d W=d u \cdot d \lambda \cdot d V=d u \cdot d \lambda \cdot d A \cdot d l$

where du is the energy density caused by radiation into the solid angle element $d \Omega$. Combining Eqs. (25) and (26) we find

$d u=\frac{1}{c} \cdot L \cdot d \Omega$.

The latter holds for any direction. The total energy density is calculated by integrating over solid angle,

$u=\frac{1}{c} \int_{4 \pi} L \cdot d \Omega=\frac{1}{c} F_{0}$.

From this calculation it is obvious that $c$ is the speed of light in the medium, rather than in vacuum.

\section{Appendix B: Absorption efficiency of a droplet in the ge- ometrical optics limit}

In the following, we calculate the absorption by a sphere with given refractive index in the geometrical optics limit. In particular, the absorbed radiant power is calculated by tracing the path of the radiation through the sphere, as outlined in Fig. 1. The total absorbed radiant power is calculated by integrating this quantity over the cross section of the sphere and over the full solid angle $4 \pi$. For this calculation it is assumed that the incident radiance $L_{0}(\theta, \phi)$ is constant over the volume of the sphere.

The angles $\alpha$ and $\beta$ are related by Snell's law of refraction

$\sin \alpha=n \cdot \sin \beta$.

At each interface, a fraction $R$ of the incident radiation is reflected, where $R$ is defined by Fresnel's equations (Kerker, 1969):

$R=\frac{1}{2}\left[\left(\frac{\sin (\alpha-\beta)}{\sin (\alpha+\beta)}\right)^{2}+\left(\frac{\tan (\alpha-\beta)}{\tan (\alpha+\beta)}\right)^{2}\right]$.

$R$ is the same for entering and exiting the medium and is valid in this form for unpolarized radiation.

Due to the spherical symmetry, the incident radiation stays in one and the same plane through the center of the sphere, and the incidence angle $\beta$ of reflection at the inner wall of the droplet is the same for all consecutive reflections. In consequence, the reflection coefficient $R$ is the same for all reflections, see Fig. 1 . The fraction of radiance initially transmitted into the sphere is

$L_{1}=L_{\text {unperturbed }} \cdot(1-R)$,

where $R$ is the reflection coefficient according to Eq. (30). 
Along each path fragment between two successive reflections, $i$ and $i+1$, the radiance is reduced by a factor $R \cdot \exp \left(-k_{\mathrm{abs}} \cdot l\right)$ where $l$ is the length of the path fragment:

$L_{\mathrm{i}+1}=L_{\mathrm{i}} \cdot R \cdot \exp \left(-k_{\mathrm{abs}} \cdot l\right)$,

where $L_{\mathrm{i}+1}$ is the radiance immediately after the $i$ 'th reflection. $R$ considers the reflection at the surface and the exponential factor considers the absorption according to LambertBeer's law. The length of the path fragment $l$ is a function of the angle $\beta$ :

$l(\beta)=2 r \sqrt{1-\sin ^{2} \beta}$.

In consequence, along each path fragment $i$ a fraction

$1-\exp \left(-k_{\mathrm{abs}} \cdot l\right)$

of the initial radiance $L_{\mathrm{i}}$ is absorbed. To calculate the fraction $f_{\text {abs }}$ of the radiance absorbed along the infinite path, the sum over all path fragments is calculated, using Eqs. (31), (32), and (34)

$$
\begin{aligned}
f_{\mathrm{abs}} \cdot & L_{\text {unperturbed }}=\sum_{i=1}^{\infty} L_{\mathrm{i}} \cdot\left[1-\exp \left(-k_{\mathrm{abs}} \cdot l\right)\right] \\
= & L_{\text {unperturbed }} \cdot(1-R) \cdot\left[1-\exp \left(-k_{\mathrm{abs}} \cdot l\right)\right] \\
& \cdot \sum_{i=1}^{\infty}\left[R \cdot \exp \left(-k_{\mathrm{abs}} \cdot l\right)\right]^{i-1} .
\end{aligned}
$$

The last term is obviously a geometrical series which can be written in closed form to finally give

$$
f_{\mathrm{abs}}=\frac{(1-R) \cdot\left[1-\exp \left(-k_{\mathrm{abs}} \cdot l\right)\right]}{1-R \cdot \exp \left(-k_{\mathrm{abs}} \cdot l\right)} .
$$

The total absorbed radiant power is calculated by integrating Eq. (35) over the cross section $A$ of the sphere and over solid angle:

$$
\begin{aligned}
\frac{d W_{\mathrm{abs}}}{d t} & =\int_{4 \pi} \int_{A} L_{\text {unperturbed }}(\theta, \phi) f_{\mathrm{abs}} d A d \Omega \\
& =\int_{A} f_{\mathrm{abs}} \int_{4 \pi} L_{\text {unperturbed }}(\theta, \phi) d \Omega d A \\
& =F_{0, \text { unperturbed }} \cdot \int_{A} f_{\mathrm{abs}} d A .
\end{aligned}
$$

The integral over the circular cross section is evaluated as follows:

$$
\begin{aligned}
\int_{A} f_{\mathrm{abs}} d A & =\int_{0}^{r} \int_{0}^{2 \pi} f_{\mathrm{abs}}(\rho) \rho d \varphi d \rho \\
& =2 \pi \int_{0}^{r} f_{\mathrm{abs}}(\rho) \rho d \rho \\
& =2 \pi r^{2} \int_{0}^{1} f_{\mathrm{abs}}(\xi) \xi d \xi
\end{aligned}
$$

with the substitution $\xi=\rho / r=\sin \alpha$.
Using Eqs. (3) and (37), the absorption efficiency can be calculated by

$Q_{\mathrm{abs}}=\frac{1}{\pi r^{2}} \int f_{\mathrm{abs}} d A$.

Combining Eqs. (35), (38), and (39) the absorption efficiency is

$Q_{\mathrm{abs}}=2 \int_{0}^{1} \frac{[1-R(\xi)] \cdot\left[1-\exp \left[-k_{\mathrm{abs}} \cdot l(\xi)\right]\right]}{1-R(\xi) \cdot \exp \left[-k_{\mathrm{abs}} \cdot l(\xi)\right]} \xi d \xi$

$R(\xi)$ is the reflection coefficient according to Eq. (30), and $l(\xi)=2 r \sqrt{1-\frac{\xi^{2}}{n^{2}}}$ is the length of a single path fragment between two reflections according to Eq. (33). Except for a factor of 2 and a missing square (which is clearly a typographical error) this is equivalent to Eq. (6) of Bohren and Barkstrom (1974) whose final results, Eqs. (9), (10), and (11) agree with our findings. Equation (40) can be evaluated numerically. However, in the special case of small absorption, $k_{\text {abs }} \cdot r \ll 1, f_{\text {abs }}$ can be replaced by its first order Taylor expansion in $k_{\text {abs }}$ :

$f_{\mathrm{abs}} \approx l(\xi) \cdot k_{\mathrm{abs}}$

and the integral can be evaluated analytically to yield

$Q_{\mathrm{abs}}=\frac{4}{3} r k_{\mathrm{abs}} n^{2} \cdot\left[1-\left(1-\frac{1}{n^{2}}\right)^{3 / 2}\right]$.

Introducing the definition of the actinic flux enhancement $\eta$ in Eq. (5), (42) evaluates to

$\eta=\frac{Q_{\mathrm{abs}} \cdot \pi r^{2}}{k_{\mathrm{abs}} \cdot V}=n^{2} \cdot\left[1-\left(1-\frac{1}{n^{2}}\right)^{3 / 2}\right]$.

This is in agreement with Eq. (9) of Bohren and Barkstrom (1974), as indicated above.

Acknowledgements. We thank M. van Weele and an anonymous reviewer for their constructive comments. B. Mayer likes to thank the German Academic Exchange service (DAAD) for funding the stay at NCAR where a major part of this research was done. NCAR is sponsored by the National Science Foundation.

Edited by: A. Hofzumahaus

\section{References}

Bohren, C. and Barkstrom, B.: Theory of the Optical Properties of Snow, J. Geophys. Res., 79, 4527-4535, 1974.

Bohren, C. and Huffman, D.: Absorption and Scattering of Light by Small Particles, John Wiley and Sons, New York, 1983.

Born, M. and Wolf, E.: Principles of Optics, 7th (expanded) edition, Cambridge University Press, 2003.

Bott, A. and Zdunkowski, W., Electromagnetic energy within dielectric spheres, J. Opt. Soc. Am. A, 4, 1361-1365, 1987. 
Chameides, W. and Davis, D.: The free radical chemistry of cloud droplets and its impact upon the composition of rain, J. Geophys. Res., 87, 4739-4755, 1982.

Chandrasekhar, S.: Radiative transfer, Oxford Univ. Press, UK, 1950.

Crawford, J., Shetter, R., Lefer, B., Cantrell, C., Junkermann, W., Madronich, S., and Calvert, J., Cloud impacts on UV spectral actinic flux observed during the International Photolysis Frequency Measurement and Model Intercomparison (IPMMI), J. Geophys. Res., 108, doi:10.1029/2002JD002 731, 2003.

Graedel, T. and Goldberg, K.: Kinetic studies of raindrop chemistry. I. Inorganic and organic processes, J. Geophys. Res., 88, $10865-$ $10882,1983$.

Hale, G. and Querry, M.: Optical Constants of Water in the 200-nm to 200- $\mu \mathrm{m}$ Wavelength-Region, Appl. Opt., 12, 555-563, 1973.

Harvey, A., Gallagher, J., and Levelt Sengers, J.: Revised formulation for the refractive index of water and steam as a function of wavelength, temperature and density, Phys. Chem. Ref. Data, 27, 761-774, 1998.

Jacob, D.: Heterogeneous chemistry and tropospheric ozone, Atmos. Env., 34, 2131-2159, 2000.

Junkermann, W.: Measurement of the $\mathrm{J}\left(\mathrm{O}^{1} D\right)$ actinic flux within and above stratiform clouds and above snow surfaces, Geophys. Res. Lett., 21, 793-796, 1994.

Kerker, M.: The Scattering of Light and Other Electromagnetic Radiation, Academic Press, New York, San Francisco, London, 1969.

Kosak-Channing, L. and Helz, G.: Solubility of ozone in aqueous solutions of $0-0.6 \mathrm{M}$ ionic strength at 5-30 C, Environ. Sci. Technol., 17, 145-149, 1983.

Lantz, K. O., Shetter, R. E., Cantrell, C. A., Flocke, S. J., Calvert, J. G, and Madronich, S.: Theoretical, actinometric, and radiometric determinations of the photolysis rate coefficient of $\mathrm{NO}_{2}$ during the Mauna Loa Observatory Photochemistry Experiment 2, J. Geophys. Res., 101, 14 613-14 629, 1996.

Lelieveld, J. and Crutzen, P.: The role of clouds in tropospheric photochemistry, J. Atmos. Chem., 12, 229-267, 1991.
Lenoble, J.: Atmospheric Radiative Transfer, A. DEEPAK Publishing, Hampton, Virginia, USA, 1993.

Los, A., Van Weele, M., and Duynkerke, P., Actinic fluxes in broken cloud fields, J. Geophys. Res., 102, 4257-4266, 1997.

Madronich, S.: Photodissociation in the atmosphere. 1. Actinic flux and the effects of ground reflections and clouds, J. Geophys. Res., 92, 9740-9752, 1987.

Mayer, B., Fischer, C., and Madronich, S.: Estimation of surface actinic flux from satellite (TOMS) ozone and cloud reflectivity measurements, Geophys. Res. Lett., 25, 4321-4324, 1998.

Mayer, B., Schröder, M., Preusker, R., and Schüller, L.: Remote sensing of water cloud droplet size distributions using the backscatter glory: a case study, Atmos. Chem. Phys., 4, 12551263, 2004,

SRef-ID: 1680-7324/acp/2004-4-1255.

Ray, A. and Bhanti, D.: Effect of optical resonances on photochemical reactions in microdroplets, Appl. Opt., 36, 2663-2674, 1997.

Ruggaber, A., Dlugi, R., and Nakajima, T.: Modelling of Radiation Quantities and Photolysis Frequencies in the Troposphere, J. Atmos. Chem., 18, 171-210, 1994.

Ruggaber, A., Dlugi, R., Bott, A., Forkel, R., Herrmann, H., and Jacobi, H.-W.: Modeling of radiation quantities and photolysis frequencies in the aqueous phase in the troposphere, Atmos. Env., 31, 3137-3150, 1997.

Wiscombe, W.: Mie Scattering Calculations: Advances in Technique and Fast, Vector-Speed Computer Codes, Tech. Rep. TN140+STR, NCAR, edited and revised 1996, 1979.

Wiscombe, W.: Improved Mie scattering algorithms, Appl. Opt., 19, 1505-1509, 1980.

Wiscombe, W.: refwat, a program to calculate the complex refractive index of pure liquid water for wavelengths between $0.01 \mathrm{mi}-$ crons and $10.0 \mathrm{~m}$, ftp://climate.gsfc.nasa.gov/wiscombe/, 1994.

Yin, Y., Parker, D., and Carslaw, K.: Simulation of trace gas redistribution by convective clouds - Liquid phase processes, Atmos. Chem. Phys., 1, 19-36, 2001,

SRef-ID: 1680-7324/acp/2001-1-19. 